\title{
Pulsation Hydrodynamics of Luminous Blue Variables and Pulsation-Driven Winds
}

\author{
Joyce A. Guzik, Arthur N. Cox, Kate M. Despain, and Michael S. Soukup \\ Los Alamos National Laboratory, Los Alamos, NM 87545-2345 USA
}

\begin{abstract}
Many physical factors, including radial and nonradial pulsation, rotation, radiation pressure, convection, magnetic fields, or dynamical instabilities may play important roles in the hydrodynamics of Luminous Blue Variables. We review the current status of hydrodynamic modeling of LBV envelopes, and describe results of our models using the one-dimensional nonlinear hydrodynamics code of Ostlie and Cox. We find that the models pulsate in several simultaneous radial modes, driven by the helium and Fe ionization $\kappa$ effect. The pulsations have quasi-periods between 5 and 80 days, with radial velocity amplitudes of $50-200 \mathrm{~km} / \mathrm{sec}$, and may be identified with the LBV microvariations. In some cases, depending on luminosityto-mass ratio and helium abundance, deep layers in the model can periodically exceed the Eddington luminosity limit. The key to exceeding $\mathrm{L}_{E}$ is the inclusion of the time dependence of convection: Near the regions of opacity peaks produced by $\mathrm{Fe}$ and helium ionization, convection is turning on and off during each pulsation cycle. If convection cannot turn on rapidly enough to transport the required luminosity through the region, the Eddington limit is exceeded. If this region of the star is sufficiently adiabatic, an "outburst" may occur. In the hydrodynamic models, an outburst is indicated by the photospheric radial velocity suddenly becoming very large, and the photospheric radius increasing monotonically over several pulsation cycles. Such pulsation-triggered outbursts may be responsible for the driving of variable, nonspherical winds. If large and infrequent enough, these outbursts may be identified with the classic LBV eruptions accompanied by episodic mass loss.
\end{abstract}

\section{Mechanisms Proposed to Destabilize LBV Envelopes}

Many mechanisms have been proposed to destabilize the envelopes of Luminous Blue Variable stars, making them susceptible to prodigious mass outflow and sporadic S Doradus-type or Eta Carinae-like outbursts. It is likely that all of these mechanisms are relevant at some stage of a massive star's evolution, or at different levels in the stellar envelope.

The envelopes of LBV stars are very close to the Eddington luminosity limit, at which the outward acceleration due to radiation pressure equals the inward gravitational acceleration. As discussed by Lamers (1997) and Langer (1997), the classical Eddington luminosity $\mathrm{L}_{\text {edd }}=4 \pi c G \mathrm{M} / \kappa_{e}$ is modified for stellar envelopes by replacing the electron scattering opacity $\kappa_{e}$ with the flux-mean opacity $\kappa$, and by reducing the gravitational acceleration by the centripetal acceleration for rotating stars. Thus the Eddington limit can vary within a stellar envelope, and as a function of latitude, and also varies 
with mass, metallicity, and evolutionary state. Lamers (1997) concludes that if a stellar envelope is within 5 or $10 \%$ of this "Atmospheric Eddington Limit", the envelope is loosely-bound enough that any small perturbation will cause the envelope to react violently. Langer (1998) shows that during main-sequence evolution of rotating massive stars, the surface layers reach the rotation-modified Eddington limit, called the $\Omega$ limit, and mass loss occurs first, and preferentially, along the equatorial plane. Angular momentum loss in the wind restricts the star's rotation rate to the $\Omega$ limit during subsequent evolution, and determines the maximum mass-loss rate, which is comparable to the observed LBV mass-loss rate.

Maeder (1989) finds that as massive stars evolve redward across the HR diagram, envelope opacities increase and the outer layers become supraEddington, resulting in a density inversion. Maeder suggests that such density inversions trigger instability, resulting in ejection of the outer layers above the density inversion. As the outer layers are ejected, the instability region moves downward into the star, with a rate characteristic of the local thermal timescale which is comparable to the dynamical timescale. Thus, the instability can encompass a significant fraction of the envelope during one episode, and result in mass loss of $0.1-1 \mathrm{M}_{\odot}$.

Stothers and Chin (1993) discuss the roles of the Fe-ionization opacity enhancement at $200,000 \mathrm{~K}$ and high radiation pressure in producing a region in the stellar envelope where $\Gamma_{1} \leq 4 / 3$, the condition for dynamical instability. They suggest that the onset of dynamical instability is responsible for the mass expulsions seen in LBV outbursts; however, the models that Stothers and Chin find dynamically unstable are far redward of the LBV instability strip and the Humphreys-Davidson limit. Appenzeller (1989) emphasizes the role of radiation pressure in extending the envelopes of LBV stars, and in driving them into a state of dynamical instability, in which additional expansion results in further mass loss. The envelope of the star recovers on a thermal timescale, until conditions for expansion and onset of instability are again achieved (the "relaxation oscillation" scenario).

Pulsation also likely plays a role in destabilizing LBV envelopes and enhancing mass loss. According to linear nonadiabatic pulsation stability analysis, nearly all of the stars in the upper HR diagram are unstable to radial and nonradial pulsations, including p-modes, g-modes, radial modes, and socalled "strange modes" (see e.g. Glatzel and Kiriakidis 1993, Glatzel 1997; Cox et al. 1997). Some modes are predicted to have large growth rates in the linear analysis. Pulsation also has the potential to extend the stellar envelope. Glatzel and Kiriakidis (1993) suggest that resonant coupling between modes could trigger an LBV outburst. As discussed by Guzik et al. (1997) and Cox et al. (1998), the variations in physical conditions and radiation-matter interactions during a pulsation cycle can cause regions of the envelope to periodically exceed $\mathrm{L}_{E}$. 


\section{Nonlinear Pulsation Hydrodynamic Modeling}

Several groups have calculated nonlinear pulsation hydrodynamic models of Stars that have some physics in common with LBVs, such as strange mode instability, and high luminosity-to-mass (L/M) ratios. Kiriakidis et al. (1997) and Glatzel et al. (1998) discuss nonlinear evolution of strange modes in massive main sequence and Wolf-Rayet stars. They find that pulsation amplitudes can reach $\sim 100 \mathrm{~km} / \mathrm{sec}$, sufficient to exceed the escape velocity and to drive mass loss in massive main-sequence stars. Preliminary hydrodynamic models of LBV envelopes by Dorfi and Feuchtinger (1998, private communication) exhibit regular pulsations, and a rearrangement of the envelope structure as kinetic energy is deposited in outer layers, but no pulsationdriven mass loss. Bono and Marconi (1998) and Buchler et al. (1998, private communication) have examined nonlinear pulsations in Cepheid variables, with particular attention to the interaction between pulsation and convection. Aikawa and Sreenivasan (1996) examine the role of strange modes and a density inversion in nonlinear pulsations of 0.6 and $4 \mathrm{M}_{\odot}$ supergiant models, and comment on differences in envelope structure produced by the strange modes, compared to typical radial modes. Heger et al. (1997) considered 10$20 \mathrm{M}_{\odot}$ red supergiant models with high $\mathrm{L} / \mathrm{M}$ ratios, and find large amplitude pulsations similar to those posited as precursors for the "superwind" of AGB stars.

Here we summarize our preliminary results for nonlinear hydrodynamic models of LBV envelopes (see also Guzik et al. 1997; Cox et al. 1997; Cox et al. 1998; Despain et al. 1998). The unique and essential ingredient in the Los Alamos models is the inclusion of a time-dependent convection treatment.

We base the composition, mass, luminosity, and effective temperature of our models on evolution models of initial mass 50 and $80 \mathrm{M}_{\odot}$, and $Z=0.01$ or 0.02 , on their first crossing to the red in the HR diagram. We vary the radius exponent in the mass-loss parametrization of Nieuwenhuijzen and de Jager (1990) to produce a range of surface helium abundance enhancements when the models reach the LBV instability region.

We next create hydrostatic envelope models with 60-120 zones, based on the evolution models. The models have extremely compact cores, and highly extended envelopes containing $95 \%$ of the stellar radius, but only $0.001 \%$ of the stellar mass. Linear nonadiabatic pulsation analysis shows that the models are unstable to pulsations driven by the $\kappa$ mechanism, primarily in the Fe-bump opacity region for massive models, and in the He-ionization region for somewhat lower-mass models. The models are also unstable to multiple radial "strange modes", that have no counterpart in adiabatic analysis. These modes have large growth rates, typically more than $100 \%$ per period, and in one case exceeding $600 \%$ per period!

As discussed by Langer (1997), in a static model, the deeper layers of a stellar envelope avoid exceeding $\mathrm{L}_{E}$ because convection turns on to transport the excess luminosity. However, we note that convective energy transport in 
reality takes some time to turn on and build up efficiency, and this time can be a significant fraction of a pulsation cycle. During this time, the Eddington limit can be exceeded. To investigate this possibility, we use an updated version of the Lagrangian nonlinear hydrodynamics code described by Ostlie (1990), Cox (1990), and Cox and Ostlie (1993). The models use OPAL opacities and equation of state. This code includes a parametrized time-dependent convection treatment, based on the mixing-length theory of convection. The convective velocity of a zone at a given timestep is determined by a weighted interpolation between convective velocities of the past two timesteps, and the instantaneous velocity from mixing-length theory. Nonlocal effects are also included via a weighted average of local convective velocity with the convective velocities of neighboring zones.

The hydrodynamic models are initiated in the most unstable radial mode predicted by linear analysis, with radial velocity amplitude one $\mathrm{km} / \mathrm{sec}$ inward. We find for all of our models that the pulsation amplitude grows rapidly. For models with sufficiently high $\mathrm{L} / \mathrm{M}$ ratio and high envelope hydrogen abundance, the outward photospheric radial velocity suddenly becomes very large, and the photospheric radius monotonically increases during several pulsation periods. Eventually the outer zones of the model reach densities and temperatures beyond the lower limits of our opacity and equation of state tables, ending the hydrodynamic simulation. We define this behavior as an "outburst".

A closer examination of the models that outburst shows $\mathrm{L}_{E}$ is exceeded in zones near the Fe-opacity bump near $200,000 \mathrm{~K}$ each pulsation cycle. Figure 1 illustrates this behavior for a convecting zone (zone 20) near $200,000 \mathrm{~K}$ of a $47.28 \mathrm{M}_{\odot}$ model, with envelope $\mathrm{Y}, \mathrm{Z}=0.02,0.38$. Convection turns on to carry nearly all of the luminosity in zone 20 at about $0.4 \times 10^{6} \mathrm{~s}$, but just before this time, the Eddington luminosity of the zone has decreased (due to increased opacity), and the radiative luminosity has increased and exceeded $\mathrm{L}_{E}$. This behavior occurs again at 0.8 and $1.6 \times 10^{6}$ seconds; however, this zone has also moved outward in radius and may no longer be the zone where $\mathrm{L}_{E}$ is exceeded by the largest amount.

For temperatures and densities typical of these LBV envelope models, the opacity decreases by about $5 \%$ for an increase in $\mathrm{Y}$ of $10 \%$. If $\mathrm{Y}$ increases sufficiently due to additional mass loss, the models can avoid exceeding $\mathrm{L}_{E}$. The hydrodynamic models then show pulsations in one or more simultaneous modes, with periods of 5-50 days, similar to the periods of observed LBV microvariations, and amplitudes of $\sim 50-150 \mathrm{~km} / \mathrm{sec}$, decreasing systematically with increasing $\mathrm{Y}$.

We need to examine the sensitivity of our results to the number and distribution of zones, amount of artificial viscosity, inclusion of turbulent pressure and energy, mixing length, time lagging and spatial averaging of convective velocity in the time-dependent convection treatment, pulsation mode of initiation, and initial radial velocity amplitude. We expect that any of 


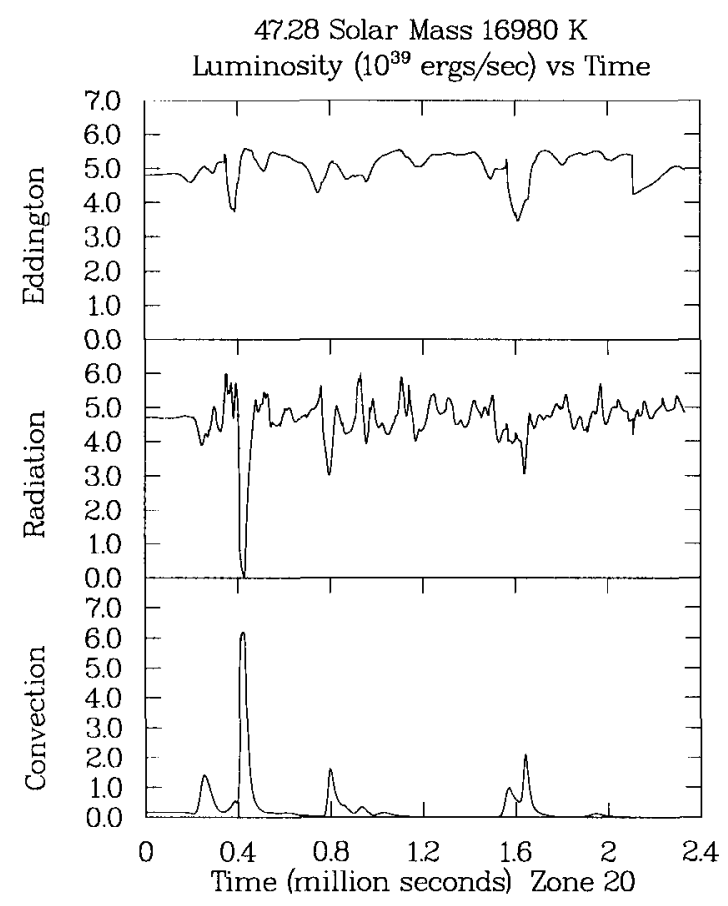

Fig. 1. Eddington, radiative, and convective luminosity of LBV model envelope zone near $200,000 \mathrm{~K}$. As the opacity due to $\mathrm{Fe}$ ionization increases during the pulsation cycle, the Eddington luminosity increases, but convection does not turn on rapidly enough to transport the luminosity, and the radiative luminosity exceeds the Eddington limit. These conditions occur at $0.4,0.8$, and 1.6 million seconds.

these may change the quantitative details, but will not destroy the conclusion that regions of the stellar envelope can briefly exceed $\mathrm{L}_{E}$ each pulsation cycle while convection is becoming established to transport some luminosity. We also need to address with a separate code, or a code with enhanced capabilities, the fate of the mass outflow, development and propagation of shocks, replenishment of the envelope by material from the core, whether the envelope structure stabilizes or becomes periodically unstable, and how the envelope recovers from an outflow event.

We have shown only that pulsations can trigger instability, and cause the outer layers of the stellar model to move outward rather than oscillate with the pulsation cycle. However, the envelopes of these models contain only $10^{-4} \mathrm{M}_{\odot}$, wherease in observed LBV outbursts much more mass can be lost. Furthermore, the predicted outflow is generated after a few pulsation cycles 
(less than one year), beginning from a near-static configuration, whereas the observed time between LBV eruptions is years or decades. Thus, we may have discovered a mechanism for a pulsation-modulated wind, whereby pulsation amplitude builds up and pushes off the outer $10^{-4} \mathrm{M}_{\odot}$ of material every few pulsation cycles. On the other hand, if Davidson's (1989) "geyser" analogy/hypothesis applies, once an instability is generated, the "trigger" may be able to propagate deeper into the star on a dynamical timescale, and involve a larger fraction of the star. In this case, this mechanism may be able to initiate substantial episodic mass ejection as is seen in LBV outbursts.

Our models do not include rotation, and treat only one-dimensional radial pulsations. Rotation reduces the effective gravitational force and modifies $\mathrm{L}_{E}$ as a function of latitude, and may result in a latitude-dependent pulsation amplitude and envelope extension, or pulsation-modulated mass loss. In addition, high-degree nonradial modes are expected in the linear analysis to have larger growth rates than radial modes (Glatzel 1997). Do these modes grow to large enough amplitudes to impose a nonspherical perturbation on the outflows?

\section{Addendum on $\eta$ Carinae}

Humphreys (these proceedings) presented the case that the most extreme LBV stars $\eta$ Car, SN 1961V, and P Cyg experienced a giant eruption, followed by a second eruption of comparable strength (but obscured by ejecta from the first eruption), with an interval of 40-50 years. If this interval corresponds to a "recovery time" between giant outbursts, and can be associated with the thermal timescale of a portion of the LBV envelope, the amount of mass involved in the outburst is very large. For example, applying this argument for $\eta \mathrm{Car}, \sim 12 \mathrm{M}_{\odot}$ participated in the eruption. This implies a deep-seated mechanism for the outbursts. It also implies that for a given amount of mass that escapes in the outburst (roughly $2 \mathrm{M}_{\odot}$ for $\eta$ Car), several times this much mass readjusts back onto the star during the recovery, and sets up the conditions for another outburst. In the case of $\eta$ Car, there is evidence that the first outburst generated mass loss in bipolar lobes at the poles, while the second generated mass outflow predominantly along the equator (N. Smith et al., these proceedings). How can we reconcile this observation with proposed mechanisms for giant outbursts?

\section{Acknowledgments:}

The authors gratefully acknowledge P. Bradley, W. Glatzel, H. Lamers, K. Oedegaard, R. Humphreys, N. Smith, J. Cassinelli, S. Owocki, S. Shore, G. Koenigsberger, N. Langer, T. Aikawa, S.R. Sreenivasan, A.M. van Genderen, G. Bono, J. Bjorkman, and C. Sterken for useful discussions. 


\section{References}

Aikawa, T., Sreenivasan, S. R. (1996): PASJ 48, 29

Appenzeller, I. (1989): in Physics of Luminous Blue Variables, ed. K. Davidson et al. (Kluwer Academic, Dordrecht), p. 195

Bono, G., Marconi, M. (1998): in A Half Century of Stellar Pulsation Interpretations, ed. P.A. Bradley and J.A. Guzik (ASP), p. 287

Cox, A. N. (1990): in Nonlinear Astrophysical Fluid Dynamics, ed. J.R. Buchler and S. T. Gottesman (New York Acad. Sciences), p. 54

Cox, A. N., Ostlie, D. A. (1993): Ap \&SS 210, 311

Cox, A. N., Guzik, J. A., Soukup, M. S., Despain, K. M. (1998): in A Half Century of Stellar Pulsation Interpretations, ed. P.A. Bradley and J.A. Guzik (ASP), p. 302

Cox, A. N., Guzik, J. A., Soukup, M. S. (1997): in Luminous Blue Variables: Massive Stars in Transition, ed. A. Nota and H.J.G.L.M. Lamers (ASP), p. 133

Davidson, K. (1989): in Physics of Luminous Blue Variables, ed. K. Davidson et al. (Kluwer Academic, Dordrecht), p. 101

Despain, K.M., Guzik, J.A., Cox, A.N. (1998): in A Half Century of Stellar Pulsation Interpretations, ed. P.A. Bradley and J.A. Guzik (ASP), p. 307

Glatzel, W., Kiriakidis, M. (1993): MNRAS 263, 375

Glatzel, W. (1997): in Luminous Blue Variables: Massive Stars in Transition, ed. A. Nota and H.J.G.L.M. Lamers (ASP), p. 128

Glatzel, W., Kiriakidis, M., Chernigovskij, S., Fricke, K.J. (1998): MNRAS, in press

Guzik, J. A., Cox, A.N., Despain, K.M., Soukup, M. S. (1997): in Luminous Blue Variables: Massive Stars in Transition, ed. A. Nota and H.J.G.L.M. Lamers (ASP), p. 138

Heger, A., Jeannin, L., Langer, N., Baraffe, I. (1997): A\&A 327, 224

Humphreys, R. (1998): these proceedings

Jeannin, L., Fokin, A.B., Gillet, D. Baraffe, I. (1997): A\&A 326, 203

Kiriakidis, M., Chernigovskij, S., Fricke, K. J., Glatzel, W. (1997): in Luminous Blue Variables: Massive Stars in Transition, ed. A. Nota and H.J.G.L.M. Lamers (ASP), p. 150

Lamers, H. J. G. L. M. (1997): in Luminous Blue Variables: Massive Stars in Transition, ed. A. Nota and H.J.G.L.M. Lamers (ASP), p. 76

Langer, N. (1997): in Luminous Blue Variables: Massive Stars in Transition, ed. A. Nota and H.J.G.L.M. Lamers (ASP), p. 83

Langer, N. (1998): A\&A 329, 551

Maeder, A. (1989): in Physics of Luminous Blue Variables, ed. K. Davidson et al. (Kluwer Academic, Dordrecht), p. 15

Nieuwenhuijzen, H. and de Jager, C. (1990): A\&A 231, 134

Ostlie, D. A. (1990): in The Numerical Modelling of Nonlinear Stellar Pulsations: Problems and Prospects, ed. J.R. Buchler (Kluwer Academic, Dordrecht), p. 89

Smith, N., Guzik, J. A., Lamers, H. J. G. L. M., Cassinelli, J. P., Humphreys, R. (1998): Blitz Model for the Eruptions of Eta Carinae, these proceedings

Stothers, R.B., Chin, C.-W. (1993): ApJ 408, L85 


\section{Discussion}

J. Puls: How sensitively do your results depend on the outer boundary condition, e.g., the presence of a wind?

A. Guzik: The models do not include a wind, but do include zones beyond the photosphere. Since the place where this mechanism operates is deep in the interior, at $200000 \mathrm{~K}$, I do not think that including a steady wind would affect the result.

N. Langer: I was surprised to hear that you expect only quantitative changes from changing the coupling between the pulsations and convection. Is this coupling not one of the fundamental uncertainties of your model which suitably chosen - could even make the "outbursts" go away?

A. Guzik: We have not explored all possibilities, but as long as convection does not adapt instantaneously to conditions in the zone, some convective zones in the model cannot avoid exceeding the Eddington limit for some fraction of each pulsation cycle.

S. Shore: These are neat results. You have put a characteristic time scale into the model because of the "switch on/off" convection scheme, so in a very non-linear model I am not surprised that you get this resonance-like behaviour. Have you explored the range of parameters $\left(\alpha=l / H_{\mathrm{p}}\right.$ and this time delay) to see if there are characteristic instability regimes?

A. Guzik: We did not tune the mixing length and time-dependent convection lag factor to find a resonance. It is a good suggestion to try varying these parameters relative to each other to see whether a resonance exists, or whether the conditions that lead to an "outburst" disappear for certain choices.

G. Koenigsberger: What determines the speed with which convection initiates in your code?

A. Guzik: The criterion for turning on convection is given by the standard mixing length theory criterion, $\nabla_{\mathrm{rad}}>\nabla_{\mathrm{ad}}$. The rate at which convection turns on in a particular zone is modified from the instantaneous values given by mixing-length theory according to the lag in time specified by the timedependent convection parameterization. The lag time is the same fraction of the convection time scale $=$ mixing length $/$ convective velocity determined by some average of the condition in the previous time steps. 